\title{
SEMI-DIVISORIALITY OF HOM-MODULES AND INJECTIVE COGENERATOR OF A QUOTIENT CATEGORY
}

\author{
HWANKOO KIM
}

\begin{abstract}
In this paper, we study $w$-nullity and (co-)semi-divisoriality of Hom-modules and the semi-divisorial envelope of $\operatorname{Hom}_{R}(M, N)$ under suitable conditions on $R, M$, and $N$. We also investigate an injective cogenerator of a quotient category.
\end{abstract}

\section{Introduction}

Let $R$ be an integral domain. In [17] Wang and McCasland defined semidivisorial closure, or $w$-closure for torsion-free $R$-modules. In [7], H. Kim extended this notion to any $R$-module and introduced and studied the related notions of co-semi-divisoriality and $w$-nullity. In $[7,8,9]$ these concepts were then used to give new module-theoretic characterizations of $t$-linkative domains, generalized GCD domains, and strong Mori domains, classes of domains widely considered in multiplicative ideal theory.

Earlier, in $[1,12,13]$, Beck, Nishi and Shinagawa investigated injective modules over a Krull domain in terms of co-divisorial modules, pseudo-null modules, and divisorial modules and investigated pseudo-nullity and (co-)divisoriality of Home-modules. In particular, it was shown that in the case of a Krull domain $R$ with quotient field $K$, the injective envelope $E(K / R)$ of $K / R$ is a cogenerator of the quotient category $\operatorname{Mod}(R) / \mathscr{M}_{0}$, where $\operatorname{Mod}(R)$ is the category of all unitary $R$-modules and $\mathscr{M}_{0}$ is the thick subcategory of the modules with trivial maps into the codivisorial modules. Recently, in [11] Mouçouf characterized the rings of Krull type $R$ with quotient field $K$ such that the (canonical) functorial image of $E(K / R)$ is an injective cogenerator of the quotient category $\operatorname{Mod}(R) / \mathscr{M}_{0}$. Also in [16], Wang investigated the case when Hom-modules are semi-divisorial in torsion-free.

Received July 31, 2009.

2010 Mathematics Subject Classification. Primary 13A15; Secondary 13D30.

Key words and phrases. (co-)semi-divisorial, w-null, cogenerator, Hom-module, Hdomain, Krull domain, torsion theory.

This research was supported by Basic Science Research Program through the National Research Foundation of Korea(NRF) funded by the Ministry of Education, Science and Technology(2010-0011996). 
In this paper, we study an injective cogenerator of a quotient category and $w$-nullity and (co-)semi-divisoriality of Hom-modules using methods developed in $[1,11,12,13]$. As a corollary, for the class of completely integrally closed domains, we characterize Krull domains in terms of an injective cogenerator of a quotient category. We also investigate the semi-divisorial envelope of $\operatorname{Hom}_{R}(M, N)$ under suitable conditions on $R, M$, and $N$.

Throughout this paper, $R$ denotes an integral domain with quotient field $K$. Let $\mathcal{F}(R)$ denote the set of nonzero fractional ideals of $R$. Recall that the function on $\mathcal{F}(R)$ defined by $A \mapsto\left(A^{-1}\right)^{-1}=A_{v}$ is a star operation called the $v$-operation, where $A^{-1}=R:_{K} A=\{x \in K \mid x A \subseteq R\}$. An ideal $J$ of $R$ is called a Glaz-Vasconcelos ideal if $J$ is a finitely generated ideal of $R$ with $J^{-1}=R$. We abbreviate this as $G V$-ideal, denoted by $J \in \mathrm{GV}(R)$. Following [17], a torsion-free $R$ module $M$ is called a $w$-module if $J x \subseteq M$ for $J \in \mathrm{GV}(R)$ and $x \in M \otimes K$ implies that $x \in M$, which is said to be semi-divisorial in [4]. For a torsion-free $R$-module $M$, Wang and McCasland defined the w-envelope of $M$ in [17] as $M_{w}=\{x \in M \otimes K \mid J x \subseteq M$ for some $J \in \mathrm{GV}(R)\}$. In particular, if $I$ is a nonzero fractional ideal, then $I_{w}=\{x \in K \mid J x \subseteq I$ for some $J \in \mathrm{GV}(R)\}$. The canonical map $I \mapsto I_{w}$ on $\mathcal{F}(R)$ is a star-operation, denoted $w$. It was shown in [17] that a prime ideal $P$ of $R$ is a $w$-ideal if and only if $P_{w} \neq R$. Therefore, all prime ideals contained in a proper $w$-ideal of $R$ are also $w$-ideals. We denote by $w$-Max $(R)$ the set of $w$-maximal ideals of $R$. It is also worth noting that $w$ distributes over (finite) intersections [17, Proposition 2.5]. For unexplained terminology and notation, we refer to $[2,3,14]$.

\section{2. w-null and (co-)semi-divisorial Hom-modules}

In [7], H. Kim introduced the notions of "co-semi-divisoriality" and " $w$ nullity" of a module as follows. Let $M$ be a module over an integral domain $R$ and let $\tau(M):=\left\{x \in M \mid(\mathcal{O}(x))_{w}=R\right\}$, where $\mathcal{O}(x):=\left(0:_{R} x\right)=\operatorname{ann}_{R}(x)$ is the order ideal of $x$. Then $\tau(M)$ is a submodule of $M . M$ is said to be co-semi-divisorial (resp., w-null) if $\tau(M)=0$ (resp., $\tau(M)=M$ ). Note that the notions of co-semi-divisoriality and $w$-nullity can be interpreted in terms of a suitable torsion theory [2, Proposition IX.6.2 and Proposition IX.6.4] (with $\mathcal{P}=w-\operatorname{Max}(R))$.

Let $R$ be an integral domain, let $\mathscr{T}_{\tau}(R)$ denote the full subcategory of $\operatorname{Mod}(R)$ consisting of all modules $M$ such that $M_{P}=0$ for all $P \in w$-Max $(R)$, and let $\mathscr{F}_{\tau}(R)$ denote the full subcategory of all $R$-modules $M$ have no subobject other than zero belonging to $\mathscr{T}_{\tau}(R)$. Finally let $\mathscr{C}_{\tau}(R)$ be the full subcategory of $\operatorname{Mod}(R)$ consisting of all co-semi-divisorial and semi-divisorial $R$ modules.

In an abelian category $\mathcal{A}$, we have the following definitions:

(a) An injective object $E$ is called an injective cogenerator if $\operatorname{Hom}_{\mathcal{A}}(M, E) \neq$ 0 for every $M \in \mathcal{A}$ that is not a zero object. 
(b) A nonempty full subcategory $\mathcal{C}$ of $\mathcal{A}$ is said to be thick if, for each short exact sequence $0 \rightarrow L \rightarrow M \rightarrow N \rightarrow 0$ in $\mathcal{A}, M$ is an object of $\mathcal{C}$ if and only if $L$ and $N$ are objects of $\mathcal{C}$. It is also called a Serre subcategory of $\mathcal{A}$.

It is clear that $\mathscr{T}_{\tau}(R)$ is a thick subcategory of $\operatorname{Mod}(R)$. Then we can now consider the quotient category $\operatorname{Mod}(R) / \mathscr{T}_{\tau}(R)$ and the canonical functor $T: \operatorname{Mod}(R) \rightarrow \operatorname{Mod}(R) / \mathscr{T}_{\tau}(R)$.

As usual, we denote by $E(M)$ the injective envelope of an $R$-module $M$. The following result will be useful later on.

Proposition 2.1. The following statements are equivalent for an $R$-module $M$.

(1) $M$ is co-semi-divisorial, i.e., $M \in \mathscr{F}_{\tau}(R)$.

(2) $\mathcal{O}(x)$ is a $w$-ideal for every element $x \in M$.

(3) $(\mathcal{O}(x))_{w} \neq R$ for every nonzero element $x \in M$.

(4) $\operatorname{Hom}_{R}(N, M)=0$ for every $w$-null $R$-module $N$.

(5) $\operatorname{Hom}_{R}(N, E(M))=0$ for every $w$-null $R$-module $N$.

Proof. The equivalences of (1), (2), (3), and (4) are given in [7, Proposition 2.6], while the equivalence of (1) and (5) follows from [6, Proposition 1.2].

Note from [17, Proposition 1.4] that the annihilator ideal of any submodule of a co-semi-divisorial module is a $w$-ideal. Recall from [1] that a module $M$ is said to be codivisorial if the annihilator of every nonzero element of $M$ is a divisorial ideal. Thus in a Krull domain, the notions of co-semi-divisoriality and codivisoriality are the same.

Recall from [16, Definition 4.5] that an $R$-module $M$ is said to be $w$-vanishing if $M_{P}=0$ for any maximal $w$-ideal $P$ of $R$.

Proposition 2.2. Let $N$ be an R-module. Then the following statements are equivalent.

(1) $N$ is $w$-null, i.e., $M \in \mathscr{T}_{\tau}(R)$.

(2) For each $x \in N, \mathcal{O}(x)$ is not contained in any maximal $w$-ideal.

(3) $N$ is w-vanishing.

(4) There is a torsion-free $R$-module $F$ with $N \cong F_{w} / F$.

(5) $\operatorname{Hom}_{R}(N, E(M))=0$ for every co-semi-divisorial $R$-module $M$.

Proof. The equivalences of (1), (2), (3), and (4) are given in [7, Proposition 9.3], while the equivalence of (1) and (5) follows from [6, Proposition 1.2].

Now we study $w$-nullity and (co-)semi-divisoriality of Hom-modules. It was shown in [7, Proposition 3.1] that an $R$-module $M$ is co-semi-divisorial if and only if $\operatorname{Hom}_{R}(\mathcal{Z}(R), M)=0$, where $\mathcal{Z}(R):=\bigoplus_{\left\{I \leq R \mid I_{w}=R\right\}} R / I$.

Proposition 2.3. Let $R$ be an integral domain and let $M$ and $N$ be $R$-modules. If $M$ is co-semi-divisorial, then so is $\operatorname{Hom}_{R}(N, M)$. 
Proof. By [7, Proposition 2.6], it suffices to show that $\operatorname{Hom}_{R}\left(L, \operatorname{Hom}_{R}(N, M)\right)$ $=0$ for every $w$-null $R$-module $L$. But this follows from $\operatorname{Hom}_{R}\left(L, \operatorname{Hom}_{R}(N, M)\right)$ $\cong \operatorname{Hom}_{R}\left(N, \operatorname{Hom}_{R}(L, M)\right)=0$ since $M$ is co-semi-divisorial.

Proposition 2.4. Let $R$ be an integral domain and let $M$ and $N$ be any $R$ module. If $M$ is $w$-null, then so is $\operatorname{Tor}_{n}^{R}(N, M)$ for all $n \geq 0$.

Proof. First we consider the case $n=0$. For every co-semi-divisorial $R$-module $L$ we have $\operatorname{Hom}_{R}\left(N \otimes_{R} M, E(L)\right) \cong \operatorname{Hom}_{R}\left(N, \operatorname{Hom}_{R}(M, E(L))\right)=0$ since $M$ is $w$-null; therefore $N \otimes_{R} M$ is $w$-null by Proposition 2.2. For the case when $n \geq 1$, we consider a projective resolution of $N$ :

$$
\cdots \rightarrow P_{n} \rightarrow P_{n-1} \rightarrow \cdots \rightarrow P_{2} \rightarrow P_{1} \rightarrow P_{0} \rightarrow N \rightarrow 0 .
$$

Then, since each $P_{i} \otimes M$ is $w$-null, we can see that $\operatorname{Tor}_{n}^{R}(N, M)$ is $w$-null for every $n \geq 0$ by noting that the submodules and homomorphic images of $w$-null modules are also $w$-null.

Now we recall some definitions from [7]: Let $M$ be an $R$-module. Then $W(M):=\pi^{-1}(\tau(E(M) / M))$ is called the semi-divisorial envelope of $M$, where $\pi: E(M) \rightarrow E(M) / M$ is the canonical projection, $M$ is said to be semidivisorial if $W(M)=M$, and $M$ is said to be weakly $w$-flat if $\operatorname{Tor}_{1}^{R}(\mathcal{Z}(R), M)=$ 0 . It is clear from the definition that every injective $R$-module is semi-divisorial. Let $N$ be an $R$-module. Then we denote $\mathcal{U}_{w}(N):=\{L \mid L$ is a submodule of $N$ such that $\left(L:_{R} x\right)_{w}=R$ for every $\left.x \in N\right\}$.

Proposition 2.5. The following statements are equivalent for an $R$-module $M$.

(1) $M$ is weakly $w$-flat.

(2) $M^{b}:=\operatorname{Hom}_{\mathbb{Z}}(M, \mathbb{Q} / \mathbb{Z})$ is semi-divisorial.

(3) $I \otimes_{R} M \rightarrow M$ is a monomorphism for all $I \in \mathcal{U}_{w}(R)$.

(4) $L \otimes_{R} M \rightarrow N \otimes_{R} M$ is a monomorphism for all $L \in \mathcal{U}_{w}(N)$.

Proof. The equivalence of (1) and (2) is given in [7, Proposition 4.3], while the equivalences of (2), (3), and (4) are given in [14, IX, Exercise 25].

Let $M$ be a semi-divisorial $R$-module and $N$ be an $R$-module. Then it was shown in [7, Corollary 3.4] that if $\operatorname{Hom}_{R}\left(\operatorname{Tor}_{1}^{R}(\mathcal{Z}(R), N), M\right)=0$, then $\operatorname{Hom}_{R}(N, M)$ is semi-divisorial.

Theorem 2.6. Let $R$ be an integral domain, $M$ be a semi-divisorial $R$-module, and $N$ be an $R$-module. Then $\operatorname{Hom}_{R}(N, M)$ is semi-divisorial if one of the following conditions is satisfied;

(i) $M$ is co-semi-divisorial;

(ii) $N$ is weakly $w$-flat.

Proof. It suffices to show that $\operatorname{Hom}_{R}\left(\operatorname{Tor}_{1}^{R}(\mathcal{Z}(R), N), M\right)=0$ by [7, Corollary $3.4]$. 
(i) Note that $R / I$ is $w$-null for every $I \in \mathcal{U}_{w}(R)$ ([7, Proposition 2.5]). Thus we have that $\operatorname{Tor}_{1}^{R}(R / I, N)$ is $w$-null for every $I \in \mathcal{U}_{w}(R)$. Now since Tor commutes with direct sums and $w$-nullity is closed under direct sums, we have $\operatorname{Tor}_{1}^{R}(\mathcal{Z}(R), N)$ is $w$-null. Therefore $\operatorname{Hom}_{R}\left(\operatorname{Tor}_{1}^{R}(\mathcal{Z}(R), N), M\right)=0$ by the cosemi-divisoriality of $M$ ([7, Proposition 2.6]).

(ii) This follows from the definition of "weakly $w$-flat".

It was shown in [5, Proposition 2.2] that for a rank one flat ideal $I \subset K$, the endomorphism $\operatorname{End}_{R}(I)(=I: I)$ of $I$ is semi-divisorial. We extend this result to any flat module in the following corollary. Note that flat $R$-modules are torsion-free (and so co-semi-divisorial) for every integral domain $R$.

Corollary 2.7. Let $R$ be an integral domain.

(1) If $M$ is a flat $R$-module, then $\operatorname{End}_{R}(M)$ is a semi-divisorial $R$-module.

(2) If $M$ is a co-semi-divisorial and semi-divisorial $R$-module, then so is $\operatorname{End}_{R}(M)$.

(3) If $M$ is co-semi-divisorial, then $M^{*}=\operatorname{Hom}_{R}(M, R)$ is semi-divisorial.

\section{Semi-divisorial equivalence}

In this section, we investigate the semi-divisorial envelope of $\operatorname{Hom}_{R}(M, N)$ under suitable conditions on $R, M$, and $N$. To do so, we need some definitions and results.

Lemma 3.1 ([15, Proposition 1.1]). Let $R$ be an integral domain and let $L \rightarrow$ $M \rightarrow N$ be an exact sequence of $R$-modules. If $L$ and $N$ are $w$-null, then so is $M$.

Let $M$ and $N$ be $R$-modules and let $f: M \rightarrow N$ be an $R$-homomorphism. Then $f$ is said to be $w$-injective (resp., $w$-surjective) if $\operatorname{ker}(f)$ (resp., coker $(f)$ ) is $w$-null. And $f$ is said to be $w$-isomorphic if $f$ is both $w$-injective and $w$ surjective.

Lemma 3.2 ([15, Lemma 1.2]). Let $R$ be an integral domain and let $f: L \rightarrow M$ and $g: M \rightarrow N$ be homomorphisms of $R$-modules. If $f$ and $g$ are $w$-injective (resp., w-surjective or $w$-isomorphic), then so is $g f$.

Theorem 3.3 ([7, Theorem 8.1]). The following statements are equivalent for an integral domain $R$.

(1) If an $R$-module $M$ is injective, then so is $\tau(M)$.

(2) $E(\tau(M))=\tau(E(M))$ for any $R$-module $M$.

(3) Let $N$ be an essential extension of $M$. If $M$ is $w$-null, then so is $N$.

(4) Let $I \leq R$ such that $I_{w} \neq R$. Then $I:_{R} a$ is a w-ideal for some $a \in R \backslash I_{w}$.

(5) If $M$ is not w-null, then $M$ has a nonzero co-semi-divisorial submodule.

(6) If $I \leq R$, then there exists an ideal $J$ of $R$ such that $J_{w}=R$ and $I=I_{w} \cap J$. 
Recall that an integral domain $R$ is said to be pseudo-t-linkative if $R$ satisfies one of the equivalent conditions of Theorem 3.3.

Proposition 3.4. Let $R$ be a pseudo-t-linkative domain with quotient field $K(\neq R)$. Let $f: M \rightarrow N$ be a homomorphism of $R$-modules and $p: M \rightarrow$ $M / \tau(M), q: N \rightarrow N / \tau(N)$ be the canonical projections.

(1) There is a unique homomorphism $f_{*}: M / \tau(M) \rightarrow N / \tau(N)$ such that $f_{*} p=q f$.

(2) If $f$ is $w$-injective, then $f_{*}$ is injective, and if $f$ is $w$-isomorphic, then so is $f_{*}$.

(3) If $f$ is w-isomorphic and $M$ is semi-divisorial, then $f_{*}$ is an isomorphism.

Proof. (1) The existence of $f_{*}$ follows from [7, Proposition 2.8] and its uniqueness is clear.

(2) Suppose first that $f$ is $w$-injective. Since $\tau(M) \subseteq f^{-1}(\tau(N))$, we have the following exact sequence

$$
0 \rightarrow \operatorname{ker}(f) \rightarrow f^{-1}(\tau(N)) \rightarrow \tau(N) .
$$

This implies, by Lemma 3.1, that $f^{-1}(\tau(N))$ is $w$-null; therefore $\tau(M)=$ $f^{-1}(\tau(N))$. Thus $f_{*}$ must be injective. If, moreover, $f$ is $w$-surjective, then $\operatorname{coker}(f)$ is $w$-null. Since the induced homomorphism of $\operatorname{coker}(f)$ to $\operatorname{coker}\left(f_{*}\right)$ is surjective, $\operatorname{coker}\left(f_{*}\right)$ must be $w$-null.

(3) Suppose that $M$ is semi-divisorial. Then $M \cong \tau(M) \oplus M / \tau(M)$ by [7, Corollary 8.9], and hence $M / \tau(M)$ is also semi-divisorial. Now the assertion follows from [7, Corollary 5.3].

It was shown in $\left[16\right.$, Proposition 2.1] that $\operatorname{Hom}_{R}(M, N)=\operatorname{Hom}_{R}\left(M_{w}, N\right)$ for a torsion-free $R$-module $M$ and a $w$-module $N$. It follows from this result that $w$, as a functor from the category of all torsion-free $R$-modules to the category of all $w$-modules, is a reflector. The following result shows that the functor $\mathrm{W}$ is a reflector from the category $\mathscr{F}_{\tau}(R)$ to the category $\mathscr{C}_{\tau}(R)$. By the $R$-dual of an $R$-module $M$ is meant the $R$-module $M^{*}=\operatorname{Hom}_{R}(M, R)$.

Proposition 3.5. Let $R$ be an integral domain and let $M, N$ be $R$-modules. Let $i$ be the canonical injection of $M$ to $W(M)$. If $N$ is co-semi-divisorial, then

$$
\operatorname{Hom}_{R}(i, W(N)): \operatorname{Hom}_{R}(W(M), W(N)) \rightarrow \operatorname{Hom}_{R}(M, W(N))
$$

is an isomorphism. In particular, we have $M^{*}=(W(M))^{*}$.

Proof. Since $N$ is co-semi-divisorial, so is $W(N)$ by [7, Proposition 2.9]. On the other hand, $W(M) / M$ is $w$-null by the definition of a semi-divisorial envelope $W$. Therefore $\operatorname{Hom}(W(M) / M, W(N))=0$, which implies that $\operatorname{Hom}_{R}(i, W(N))$ is an injection. By [7, Proposition 3.2], we can see that $\operatorname{Hom}_{R}(i, W(N))$ is a surjection. 
Corollary 3.6. Let $R$ be a pseudo-t-linkative domain with quotient field $K(\neq$ $R)$. Let $f: M \rightarrow N$ be a homomorphism of $R$-modules. Then there exists a unique homomorphism $T(f): T(M) \rightarrow T(N)$ such that $T(f) i=j f$, where $i$ (resp., $j$ ) is the canonical homomorphism of $M$ (resp., $N)$ to $T(M)$ (resp., $T(N))$. Moreover, if $f$ is a w-isomorphism, then $T(f)$ is an isomorphism.

Proof. The homomorphism $f$ induces the homomorphism $f_{*}$ of $M / \tau(M)$ to $N / \tau(N)$ by Proposition 3.4. Applying Proposition 3.5 to $f_{*}$, we can obtain a homomorphism $T(f): T(M) \rightarrow T(N)$ such that $T(f) i=j f$.

It is easy to show that, similarly to the proof of Proposition 3.5, $\operatorname{Hom}(i, T(N))$ is an injection. This shows the uniqueness of $T(f)$.

Suppose now that $f$ is a $w$-isomorphism. Then by Proposition $3.4, f_{*}$ is a $w$-isomorphism $\left(f_{*}\right.$ is necessarily injective). Since the canonical injection of $M / \tau(M)$ to $T(M)$ is an essential extension, $T(f)$ must be an injection. Since both $f_{*}$ and the canonical injection of $N / \tau(N)$ to $T(N)$ are $w$-surjective, so is the composition of them by Lemma 3.2. We can conclude from this fact that $T(f)$ is a $w$-surjection. Since a $w$-isomorphism of co-semi-divisorial and semi-divisorial modules is an isomorphism by [7, Corollary 5.3], $T(f)$ must be an isomorphism.

It was shown in $\left[16, \text { Proposition 2.3] that } \operatorname{Hom}_{R}(M, N)\right)_{w}=\operatorname{Hom}_{R}\left(M, N_{w}\right)$ for a torsion-free finitely generated $R$-module $M$ and a torsion-free $R$-module $N$. As a corollary, Wang obtained that $\left(\operatorname{End}_{R}(M)\right)_{w}=\operatorname{End}_{R}\left(M_{w}\right)$ for a torsion-free finitely generated $R$-module $M$ ([16, Corollary 2.4]).

Theorem 3.7. Let $R$ be a pseudo-t-linkative domain. Let $M$ and $N$ be co-semidivisorial $R$-modules. If $M$ is a submodule of a finitely generated $R$-module $L$, then we have

$$
W\left(\operatorname{Hom}_{R}(M, N)\right) \cong \operatorname{Hom}_{R}(W(M), W(N)) .
$$

Proof. By Proposition 3.5, we have only to prove

$$
W\left(\operatorname{Hom}_{R}(M, N)\right) \cong \operatorname{Hom}_{R}(M, W(N)) .
$$

Consider the following exact sequence

$$
0 \rightarrow \operatorname{Hom}_{R}(M, N) \rightarrow \operatorname{Hom}_{R}(M, W(N)) \rightarrow \operatorname{Hom}_{R}(M, W(N) / N) .
$$

Since $N$ is co-semi-divisorial, so is $W(N)$; thus, by Proposition $2.3, \operatorname{Hom}_{R}(M$, $N)$ and $\operatorname{Hom}_{R}(M, W(N))$ are co-semi-divisorial. Also we have that $\operatorname{Hom}_{R}(M$, $W(N))$ is semi-divisorial by Theorem 2.6. Since a $w$-isomorphism of co-semidivisorial modules is an essential extension, it suffices to show that $\operatorname{Hom}_{R}(M$, $W(N) / N)$ is $w$-null.

In general, for a submodule $M_{1}$ of a finitely generated $R$-module $M_{2}$ and a $w$-null $R$-module $N_{1}$, we will show that $\operatorname{Hom}_{R}\left(M_{1}, N_{1}\right)$ is $w$-null. Set $N_{2}:=$ $E\left(N_{1}\right)$. Then $N_{2}$ is $w$-null by [7, Theorem 8.1], since $R$ is pseudo-t-linkative. Let $\left\{x_{1}, \ldots, x_{n}\right\}$ be a system of generators of $M_{2}$ and let $f \in \operatorname{Hom}_{R}\left(M_{2}, N_{2}\right)$. Then $\mathcal{O}(f)=\mathcal{O}\left(f\left(x_{1}\right)\right) \cap \cdots \cap \mathcal{O}\left(f\left(x_{n}\right)\right)$. Since each $\left(\mathcal{O}\left(f\left(x_{i}\right)\right)\right)_{w}=R$, we 
have $(\mathcal{O}(f))_{w}=R$ by the distributivity of the star-operation $w$ over finite intersection. Hence $\operatorname{Hom}_{R}\left(M_{2}, N_{2}\right)$ is $w$-null. Therefore, $\operatorname{Hom}_{R}\left(M_{1}, N_{2}\right)$ is $w$ null, since it is a homomorphic image of $\operatorname{Hom}_{R}\left(M_{2}, N_{2}\right)$. Thus $\operatorname{Hom}_{R}\left(M_{1}, N_{1}\right)$ is $w$-null since it is isomorphic to a submodule of $\operatorname{Hom}_{R}\left(M_{1}, N_{2}\right)$.

Corollary 3.8. Let $R$ be a pseudo-t-linkative domain with quotient field $K(\neq$ $R$ ) and let $M$ and $N$ be co-semi-divisorial and semi-divisorial $R$-modules. If $M$ is a submodule of a finitely generated $R$-module, then $\operatorname{Hom}_{R}(M, N)$ is semidivisorial.

Let $M$ and $N$ be an $R$-modules. We say that $M$ is semi-divisorially equivalent to $N$ if there exists a $w$-isomorphism of $W(M)$ to $W(N)$.

Proposition 3.9. Let $R$ be a pseudo-t-linkative domain with quotient field $K(\neq R)$. Let $M$ and $N$ be $R$-modules.

(1) $M$ is semi-divisorially equivalent to $N$ if and only if $W(M / \tau(M))$ is isomorphic to $W(N / \tau(N))$. In particular, the "semi-divisorial equivalence" is an equivalence relation.

(2) If $M$ is w-isomorphic to $N$, then $M$ is semi-divisorially equivalent to $N$.

Proof. (1) The necessity follows from the facts that $W(M) \cong W(\tau(M)) \oplus$ $W(M / \tau(M))$ and $W(N) \cong W(\tau(N)) \oplus W(N / \tau(N))$ by [7, Corollary 8.9] and $W(\tau(M))$ and $W(\tau(N))$ are $w$-null by [7, Theorem 8.1] since $R$ is pseudo- $t$ linkative. The sufficiency follows from Proposition 3.4.

(2) The assertion follows immediately from Corollary 3.6.

\section{Injective cogenerator of a quotient category}

In this section, we generalize some results of $[1,11]$ related to an injective cogenerator in a quotient category. We recall from [4] that a domain $R$ is said to be an $H$-domain if every ideal $I$ of $R$ with $I^{-1}=R$ is quasi-finite (i.e. $I^{-1}=J^{-1}$ for some finitely generated subideal $J$ of $I$ ).

Theorem 4.1. Let $R$ be an $H$-domain with quotient field $K(\neq R)$, and let $M$ be any $R$-module. Then $M$ is w-null if and only if $\operatorname{Hom}_{R}(M, E(K / R))=0$.

Proof. $(\Rightarrow)$ : This follows from Proposition 2.1 since $E(K / R)$ is co-semi-divisorial by [7, Corollary 2.11].

$(\Leftarrow)$ : Suppose that $M$ is not $w$-null and let $N=M / \tau(M)$. By Proposition 2.1 and $[7$, Proposition 2.8], there is a non-zero element of $x \in N$ such that $\mathcal{O}(x)$ is a proper $w$-ideal and hence $R: \mathcal{O}(x) \supsetneqq R$ (since $R$ is an H-domain). Let $a \in R: \mathcal{O}(x) \backslash R$. Then $R: R \quad a \supset \mathcal{O}(x)$. Let $f: R \rightarrow K / R$ be the homomorphism defined by $f(b)=\overline{a b}$, where $\overline{a b}$ is the class of $a b$ in $K / R$. Since $\operatorname{ker}(f)=R:_{R} a \supset \mathcal{O}(x)$, there is a non-zero homomorphism $g: R / \mathcal{O}(x) \rightarrow$ $K / R$ such that $f=g p$, where $p$ is the canonical projection of $R$ to $R / \mathcal{O}(x)$. Let $i$ be the canonical injection of $R / \mathcal{O}(x)(\cong R x)$ to $N$. Then there is a nonzero homomorphism $h$ of $N$ to $E(K / R)$ such that $i g=h j$, and hence $h q$ is a 
non-zero homomorphism of $M$ to $E(K / R)$, where $q$ is the canonical projection of $M$ to $N$.

Since $K / R \in \mathscr{F}_{\tau}(R)$, i.e., $K / R$ has no subobject other than zero belonging to $\mathscr{T}_{\tau}(R)$, then $T(E(K / R))$ is the injective envelope of the object $T(K / R)$ of $\operatorname{Mod}(R) / \mathscr{T}_{\tau}(R)$.

Corollary 4.2. If $R$ is an H-domain, then $T(E(K / R))$ is an injective cogenerator in the quotient category $\operatorname{Mod}(R) / \mathscr{T}_{\tau}(R)$. Hence every co-semi-divisorial and semi-divisorial module over an H-domain can be embedded in an injective module.

Proof. Let $T(N) \in \operatorname{Mod}(R) / \mathscr{T}_{\tau}(R)$ with $\operatorname{Hom}_{\operatorname{Mod}(R) / \mathscr{T}_{\tau}(R)}(T(N), T(E(K / R)))$ $=0$. Then by $\left[11\right.$, Lemma 2.6] we have $\operatorname{Hom}_{\operatorname{Mod}(R)}(N, E(K / R))=0$, and by Theorem 4.1 we have $N \in \mathscr{T}_{\tau}(R)$, and then $T(N)=0$. It is clearly seen that $T(E(K / R))$ is a cogenerator object of $\operatorname{Mod}(R) / \mathscr{T}_{\tau}(R)$. The last assertion follows from [14, Proposition I.6.6].

Lemma 4.3. Let $R$ be an integral domain, let $P \in w-\operatorname{Max}(R)$, let $M$ be a co-semi-divisorial $R$-module and let $f: R / P \rightarrow M$ a homomorphism. Then either $f \equiv 0$ or $f$ is injective.

Proof. Suppose that $f \not \equiv 0$ and let $f(\overline{1})=x$. Then we have $x \in M$. Since $M$ is co-semi-divisorial, then $\mathcal{O}(x)$ is a $w$-ideal, and since $x \neq 0$, there exists $Q \in w$-Max $(R)$ such that $\mathcal{O}(x) \subseteq Q$, but since $P \subseteq \mathcal{O}(x)$, we have $P \subseteq Q$ and hence $P=Q$, so $\mathcal{O}(x)=P$ and $f$ is injective.

We recall from $\left[10\right.$, III.1.4] two facts related to $\mathscr{C}_{\tau}(R), \operatorname{Mod}(R) / \mathscr{T}_{\tau}(R)$, and $T$.

(a) The subcategory $\mathscr{C}_{\tau}(R)$ of $\operatorname{Mod}(R)$ may be identified with $\operatorname{Mod}(R) / \mathscr{T}_{\tau}(R)$.

(b) Let $M$ be an $R$-module. Then $T(M)=W(M / \tau(M))$.

Therefore, we have that $T(E(K / R))=W(E(K / R) / \tau(E(K / R))) \cong E(K / R)$.

Theorem 4.4. Let $R$ be an integral domain with quotient field $K$ satisfying $\left(R:_{R} x\right)_{v}=\left(R:_{R} x\right)$ for every $x \in K$. If $T(E(K / R))$ is an injective cogenerator in the quotient category $\operatorname{Mod}(R) / \mathscr{T}_{\tau}(R)$, then $R$ is an $H$-domain.

Proof. Note that if $R$ satisfies that $\left(R:_{R} x\right)_{v}=\left(R:_{R} x\right)$ for every $x \in K$, then $K / R$ is co-divisorial. Suppose that $R$ is not an H-domain. Then by [17, Proposition 5.7] there exists a prime ideal $P$ which is $w$-maximal but not a $v$-ideal. First we show that the module $R / P$ can not be injected in $E(K / R)$. If this were not so, then the kernel of the composition $R \stackrel{\Pi}{\rightarrow} R / P \rightarrow E(R / K)$ is $P$, where $\Pi$ is the canonical projection. Then by [1, Corollary 1.7] $P$ is a $v$-ideal, which is a contradiction. Thus by Lemma $4.3, \operatorname{Hom}_{\operatorname{Mod}(R)}(R / P, E(K / R))=0$. So $\operatorname{Hom}_{\operatorname{Mod}(R) / \mathscr{T}_{\tau}(R)}(T(R / P), T(E(K / R))) \cong \operatorname{Hom}_{\operatorname{Mod}(R)}(W(R / P), E(K / R)) \cong$ $\operatorname{Hom}_{\operatorname{Mod}(R)}(R / P, E(K / R))=0$ (note that the last isomorphism follows from Proposition 3.5). Since $T(E(K / R))$ is a cogenerator object in $\operatorname{Mod}(R) / \mathscr{T}_{\tau}(R)$, 
$T(R / P)=0$, and thus $R / P \in \mathscr{T}_{\tau}(R)$, i.e., $R / P$ is $w$-null. Hence $P_{w}=R$, which is a contradiction. Therefore $R$ is an $H$-domain.

It is well known that if $R$ is a completely integrally closed domain, then $R$ satisfies the hypothesis of Theorem 4.4. Now the following result follows from Corollary 4.2, Theorem 4.4, and the fact that an integral domain $R$ is a Krull domain if and only if $R$ is a completely integrally closed H-domain ([4, 3.2(d)]).

Corollary 4.5. Let $R$ be a completely integrally closed domain. Then $R$ is a Krull domain if and only if $E(K / R)$ is an injective cogenerator in the quotient category $\operatorname{Mod}(R) / \mathscr{T}_{\tau}(R)$.

Let $M$ be any $R$-module. We have a canonical mapping:

$$
\lambda_{M}: M \rightarrow \operatorname{Hom}_{R}\left(\operatorname{Hom}_{R}(M, E(K / R)), E(K / R)\right) .
$$

Let $f \in \operatorname{Hom}_{R}(M, E(K / R))$. Then define $\lambda_{M}(m)$ by the equation $\lambda_{M}(m)(f)=$ $f(m)$ for all $m \in M$.

Theorem 4.6. Let $R$ be an $H$-domain with quotient field $K(\neq R)$, and let $M$ be any $R$-module. Then $M$ is co-semi-divisorial if and only if $\lambda_{M}$ is injective.

Proof. $(\Leftarrow)$ : This follows from the facts that $E(K / R)$ is co-semi-divisorial and $\operatorname{Hom}_{R}(L, N)$ is co-semi-divisorial whenever $N$ is co-divisorial.

$(\Rightarrow)$ : Let $x \in M \backslash\{0\}$. Since $R x$ is not $w$-null, we can find a homomorphism $f: R x \rightarrow E(K / R)$ such that $f(x) \neq 0$ by Theorem 4.1. Since $E(K / R)$ is injective, we can lift $f$ to a mapping $\bar{f}: M \rightarrow E(K / R)$. This shows that $\lambda_{M}$ is injective, since $\lambda_{M}(x)(\bar{f})=\bar{f}(x)=f(x) \neq 0$ and hence $\lambda_{M}(x) \neq 0$.

\section{References}

[1] I. Beck, Injective modules over a Krull domain, J. Algebra 17 (1971), 116-131.

[2] L. Fuchs and L. Salce, Modules over Non-Noetherian Domains, Mathematical Surveys and Monographs 84, AMS, Providence, RI, 2001.

[3] R. Gilmer, Multiplicative Ideal Theory, Queen's Papers in Pure and Applied Mathematics, 90, Queen's University, Kingston, Ontario, 1992.

[4] S. Glaz and W. V. Vasconcelos, Flat ideals II, Manuscripta Math. 22 (1977), no. 4, $325-341$.

[5] _ Flat ideals III, Comm. Algebra 12 (1984), no. 1-2, 199-227.

[6] J. S. Golan, Localizations of Noncommutative Rings, Marcel Dekker, New York, 1975.

[7] H. Kim, Module-theoretic characterizations of t-linkative domains, Comm. Algebra, 36 (2008), no. 5, 1649-1670.

[8] _ Module-theoretic characterizations of generalized GCD domains, Comm. Algebra, 38 (2010), no. 2, 759-772.

[9] H. Kim, E. S. Kim, and Y. S. Park, Injective modules over strong Mori domains, Houston J. Math. 34 (2008), no. 2, 349-360.

[10] J. L. B. Montero, B. T. Jover, and A. Verschoren, Local Cohomology and Localization, Pitman Research Notes in Mathematics Series, 226, Longman Scientific \& Technical, London, 1989.

[11] M. Mouçouf, Some results on injective modules over a ring of Krull type, Comm. Algebra 33 (2005), no. 11, 4125-4133. 
[12] M. Nishi and M. Shinagawa, Codivisorial and divisorial modules over completely integrally closed domains. I, Hiroshima Math. J. 5 (1975), no. 2, 269-292.

[13] Codivisorial and divisorial modules over completely integrally closed domains. II, Hiroshima Math. J. 5 (1975), no. 3, 461-471.

[14] B. Stenström, Rings of Quotients: An Introduction to Methods of Ring Theory, Springer-Verlag, New York, 1973.

[15] R. G. Swan, Algebraic K-theory, Lecture notes in Math. 76, Springer-Verlarg, New York, 1968.

[16] F. Wang, On w-projective modules and w-flat modules, Algebra Colloq. 4 (1997), no. 1, 111-120.

[17] F. Wang and R. L. McCasland, On w-modules over strong Mori domains, Comm. Algebra 25 (1997), no. 4, 1285-1306.

Department of InFormation SECURITy

Hoseo University

ASAN 336-795, KOREA

E-mail address: hkkim@hoseo.edu 